\title{
Erratum: Evolution of CTE- temperature curves of HCP with hydration time
}

\section{Dongxing Xuan and Zhonghe Shui}

An error appeared in this paper when it was published in Advances in

Cement Research 24(4): 171-174, http://dx.doi.org/10.1680/

adcr.2011.23.4.171.

The second author's name was misspelt. The correct presentation is shown above. 